\title{
The geometry of map equations for Trochoids
}

\author{
Sibel Pasali Atmaca \\ Muugla Sitki Kocman University, Faculty of Science, Department of Mathematics, Mugla, Turkey
}

Received: 3 February 2018, Accepted: 14 March 2018

Published online: 20 March 2018.

\begin{abstract}
We will study trochoids and related curves using the representation of these curves as mapping of the unit circle in the complex plane. Points on the unit circle, or turns, an their uses in representing curves will be introduced and developed. Then we will prove several results which illustrate properties of trochoids.
\end{abstract}

Keywords: Map equations, Trochoids, rosettes.

\section{Introduction}

Back around 1920 a series of papers by W. F. Rigge $[6,7,8,9,10]$ appeared in the American mathematical Monthly describing various ways certain bounded closed curves, including cardioids and roses, could be drawn. Rigge's work extended earlier work by R. E. Moritz [5], and both described and built machines that would produce the curves under discussion. Rigge's machine still exists in the care of the Physics Department at Creighton University in Omaha. The basic kinds of curves studied by Moritz and Rigge are those that result from harmonic motion along a line combined with uniform angular motion of the line.

The recent paper by L. M. Hall [4] in the College Mathematics Journal used the computer algebra system Mathematica to study the kinds of curves produced by the Spirograph drawing sets, which also include cardoids and roses. Mathematically, these curves are epitrochoids and hypoctrochoids, which are defined below. Many famous curves of mathematics turn out to be achievable using the Spirograph. These include the ellipse, the deltoid, the astroid, the cardoid, the nephroid, and the standard polar coordinate roses. Going beyond the Spirograph, many interesting curves can be produced by using linear combinations of the epitrochoids and hypotrochoids. Such curves can be represented parametrically using trigonometric functions, and can be easily graphed using a computer. The more recent results can also be found in $[1,2,3,11]$.

In this study, the curves will be studied as mappings of the unit circle in complex plane. Backgrounf from the paper of W.B. Carver [1] will be given in Section 2, where we introduce the basic ideas and notation. Then, in Section 3, we will use the complex mapping approach to get new proofs of some of the results of Hall [4]. Examples will be given to illustrate most of the results.

\section{Map equations and turns}

A different approach to the straight line will illustrate methods to be used later in our treatment of curves. One of the concepts is the idea of a rotation about the origin, which we will call a turn.

Definition 1. A complex number of the form $e^{i \theta}$, with absolute value unity, will be called a turn, and will usually represented by the letter $t$. 
A turn corresponds to a point on the unit circle or to a vector of unit length. Since $\sqrt{t \bar{t}}=1, \bar{t}=\frac{1}{t}$, i.e., the conjugate of a turn is its reciprocal; and, conversely, if $\bar{a}=\frac{1}{a}$, then a and $\bar{a}$ are turns. The product of two or more turns is a turn, and if $t$ is a turn then $t^{n}$ is a turn for any real $\mathrm{n}$. The quotient of any number by its conjugate is a turn.

Definition 2. By the reflex point of a line we shall mean the reflection of the origin in the line. If the line goes through the origin, the reflex point is the origin itself.

Definition 3. For the self-conjugate equation of the line $c x+y=c r$, the turn $c$ is called the clinant of the line.

The clinant gives the orientation of the line in somewhat the same way as the slope gives the orientation of a line in the Cartesian system.

The following are relations between the slope $m$ of a line in the Cartesian $\operatorname{system}(m$ is a real $)$ and the clinant $c$ of the same line in the conjugate system ( $c$ is a turn):

$$
m=\frac{i(c+1)}{c-1}, \quad c=\frac{m+i}{m-i}, \arctan m=\frac{i}{2} \log (-c) .
$$

Definition 4. By the directed angle $\phi_{12}$ from a line $\ell_{1}$ to a line $\ell_{2}$ we shall mean any angle through which the line $\ell_{1}$ may be turned to bring it into parallelism with the line $\ell_{2}$.

Lemma 1. The clinant of the line through the points $\left(x_{1}, y_{1}\right)$ and $\left(x_{2}, y_{2}\right)$ is $c=-\frac{y_{1}-y_{2}}{x_{1}-x_{2}}$.

Definition 5. The map equation of the straight line is an example of a kind of representation which can be used advantageously in the study of certain other rational (unicursal) curves.

Suppose the complex variable $x$ is equated to a rational function of a variable turn $t$,

$$
x=R(t)=\frac{P(t)}{Q(t)},
$$

where $P(t)$ and $Q(t)$ are relatively prime polynomials. As the point $t$ runs around the unit circle the point $x$ describes a rational curve. Equation (1) implies the conjugate equation

$$
y=\bar{R}\left(\frac{1}{t}\right),
$$

and equations (1) and (2) together are parametric equations of the curve. There may be turn values of $t$ for which $Q(t)=0$, and such values of $t$ would not give points of the curve. Except for such values of $t, R(t)$ and $\bar{R}\left(\frac{1}{t}\right)$ are analytic functions of $t$. Eliminating $t$ algebraically from (1) and (2), we obtain an equation of the form

$$
f(x, y)=0
$$

and it can be shown that this equation will always be self-conjugate. It will be satisfied by all points obtained by giving $t$ turn values in (1) and (2), but may sometimes be satisfied also by other points not given by the parametric equations.

Suppose that the map equation of a curve is $x=R(t)=\frac{P(t)}{Q(t)}$ and that $t_{1}$ and $t_{2}$ are two distinct values of the turn $t$, with $Q\left(t_{1}\right) \neq 0$, and hence $Q\left(t_{2}\right) \neq 0$ when $t_{2}$ is sufficiently close to $t_{1}$; and let $\left(x_{1}, y_{1}\right)$ and $\left(x_{2}, y_{2}\right)$ be the two corresponding points on the curve. The clinant of the secant line through these points is

$$
c=-\frac{y_{1}-y_{2}}{x_{1}-x_{2}}=-\frac{\frac{y_{1}-y_{2}}{t_{1}-t_{2}}}{\frac{x_{1}-x_{2}}{t_{1}-t_{2}}}
$$

If now $t_{1}$ is fixed and $t_{2}$ is made to approach $t_{1}$ taking on only turn values, the clinant of the secant will approach the clinant of the tangent to the curve at point $\left(x_{1}, y_{1}\right)$, and the fractions $\frac{y_{1}-y_{2}}{t_{1}-t_{2}}$ and $\frac{x_{1}-x_{2}}{t_{1}-t_{2}}$ will approach limits, the values of 
the formal derivatives $D_{t} y$ and $D_{t} x$ for $t=t_{1}$. Thus provided $D_{t} x \neq 0$ when $t=t_{1}$, the clinant of the tangent to the curve at the point $\left(x_{1}, y_{1}\right)$ is a value of the turn $t t_{i}$

$$
c=-\frac{D_{t} y}{D_{t} x}
$$

If either $D_{t} y$ or $D_{t} x$ is zero for $t=t_{1}$, the other is also. For with $x=R(t)$ we may write $D_{t} x=R^{\prime}(t)$. Then $y=\bar{R}\left(\frac{1}{t}\right)$ and

$$
D_{t} y=\bar{R}^{\prime}\left(\frac{1}{t}\right)\left(-\frac{1}{t^{2}}\right)
$$

Since $-\frac{1}{t^{2}}$ can not vanish, $D_{t} y$ vanishes when and only when $\bar{R}^{\prime}\left(\frac{1}{t}\right)=0$. But $\bar{R}^{\prime}\left(\frac{1}{t}\right)=0$ is the conjugate of $R^{\prime}(t)=D_{t} x$, and hence $D_{t} y$ vanishes when and only when $D_{t} x$ vanishes. This will occur only at certain singular points of the curve, and at such points the formula (4) is meaningless. Because of the relation

$$
D_{t} y=-\frac{\overline{D_{t} x}}{t^{2}}
$$

formula (4) may be written

$$
c=\frac{\overline{D_{t} x}}{t^{2} D_{t} x}
$$

a useful form which enables us to obtain the clinant of the tangent from the map equation (1) without writing the conjugate equation (2).

\section{Trochoids and Rosettes}

Definition 6. If two tangent circles have their centers on the same side of the common tangent line, and one circle remains fixed while the other is rolled around it without slipping, a hypotrochoid is traced by any point on a diameter or extended diameter of the rolling circle. If the tracing point is on the circumference of the rolling circle, the curve traced is a hypocycloid.

Definition 7. If two tangent circles have their centers on opposite sides of the common tangent line, and one circle remains fixed while the other is rolled around it without slipping, an epitrochoid is traced by any point on a diameter or extended diameter of the rolling circle. If the tracing point is on the circumference of the rolling circle, the curve traced is a epicycloid.

The general term trochoid will be used to refer to either a hypotrochoid or an epitrochoid. Assume the center of the fixed circle is at the origin. If $\theta$ is the counterclockwise angle from the positive $\mathrm{x}$-axis to the line joining the centers of the two circles, $n$ is the radius of the fixed circle, $m$ the radius of the rolling circle, $r m$ the distance from the center of the rolling circle to the tracing point, and $a$ the initial polar angle of the tracing point with respect to the center of the fixed circle, the parametric equations are easily derived.

Let us think about the fundamental period for the map equation $h[\tau, n, m, r, a]$. We have

$$
x+i y=\left[(n-m) e^{i \theta}+\frac{r m}{e^{i\left(\frac{n-m}{m}\right) \theta}}\right] e^{i a} .
$$

For $\tau=e^{i \theta}$, because the period is $2 \pi m$, to complete the curve requires $m$ times around the unit circle. But the map equation maps the unit circle (once around) to a curve, and we can't force more than one circuit. So, if the $\theta$ period is greater than $2 \pi$, the map equation will produce an incomplete curve. We want one time around the unit circle to give the complete curve, so we have to substitute for the turn $\tau$ a new turn, $t=\tau^{\frac{n-m}{m}}$. Hence,

$$
h[\tau, n, m, r, a]=\left((n-m) \tau+\frac{m r}{\tau^{\frac{n-m}{m}}}\right) e^{i a}
$$


and after the substitution, our new map equation for hypotrochoids is:

$$
\text { hymap }[t, n, m, r, a]=\left((n-m) t^{\frac{m}{n-m}}+\frac{m r}{r}\right) e^{i a},
$$

which will produce the complete curve as $t$ takes on unit circle values one time each.

Similarly, substituting $t=\tau^{\frac{n+m}{n}}$, we define the epitrochoid map equation:

$$
\text { epmap }[t, n, m, r, a]=\left((n+m) t^{\frac{m}{n+m}}-r m t\right) e^{i a} .
$$

We will use the functions hymap and epmap to study trochoids from the map equation point of view.

For example, $h[\tau, 3,2,1,0]$ doesn't produce a complete curve as we see in Figure 3.

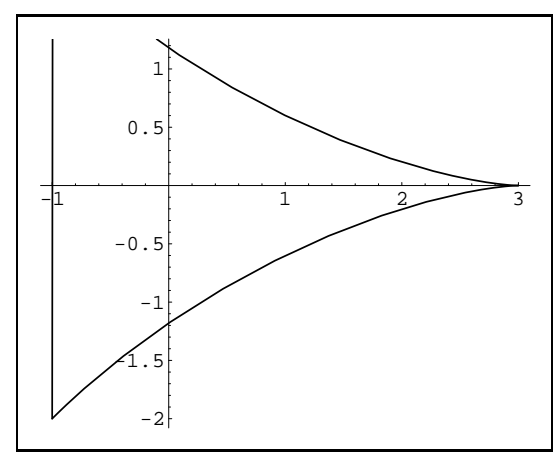

Fig. 1: hymap $[\tau, 3,2,1,0]$.

Substituting $t=\tau^{1 / 2}$, we get the equation

$$
\operatorname{hymap}[t, 3,2,1,0]=t^{2}+\frac{2}{t}
$$

for which one full period is produced as shown in Figure 2. Map equations are simpler than parametric equations. It is easier to study many of the properties of trochoids and their proofs using these map equations.

Theorem 1. If $(n, m)=1$, then

(i) hymap $[t ; x n, x m, r, a]=x$ hymap $[t ; n, m, r, a]$.

(ii) epmap $[t ; x n, x m, r, a]=x$ epmap $[t ; n, m, r, a]$.

Proof. Suppose $n$ and $m$ are relatively prime. Then,

$$
\text { hymap }[t ; x n, x m, r, a]=\left[x(n-m) t^{\frac{m}{n-m}}+\frac{x m r}{t}\right] e^{i a}=x\left[(n-m) t^{\frac{m}{n-m}}+\frac{m r}{t}\right] e^{i a}=x \text { hymap }[t ; n, m, r, a] .
$$

Similarly, we can show (ii).

A classical result regarding trochoids is that any hypotrochoid can be expressed in terms of an epitrochoid and vice-versa. This is sometimes referred to as the Bernoulli - Euler- Goldbach double generation theorem, and can be proved using the hymap and epmap functions.

Theorem 2. (The B-E-G Theorem) Let $n$ and $m$ be nonzero integers, and $r$ be nonzero. Then

(i) epmap $[t, n, m, r, a]=r$ hymap $\left[\tau, n, n+m, \frac{1}{r}, a\right]$, where $\tau=t^{-\frac{m}{n+m}}$. and

(ii) hymap $[t, n, m, r, a]=r \operatorname{epmap}\left[\tau, n, m-n, \frac{1}{r}, a\right]$ where $\tau=t^{\frac{m}{m-n}}$. 


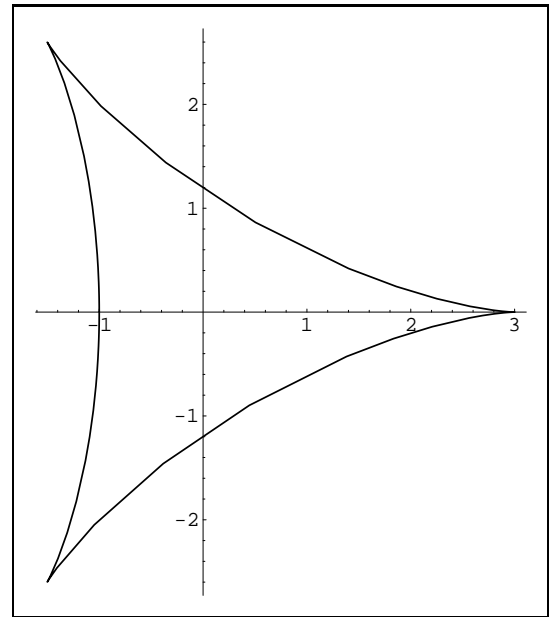

Fig. 2: hymap[t,3,2,1,0].

Proof. (i) Let epmap $[t, n, m, r, a]=\left((n+m) t^{\frac{m}{n+m}}-r m t\right) e^{i a}$. Let $\tau=t^{-\frac{m}{n+m}}$. Then $t=\tau^{-\frac{n+m}{m}}$. We get

$$
\begin{aligned}
\operatorname{epmap}[t, n, m, r, a] & =\left[(n+m) t^{\frac{m}{n+m}}-r m t\right] e^{i a} \\
& =r\left[(n+m) \frac{1}{r} t^{\frac{m}{n+m}}-m t\right] e^{i a} \\
& =r\left[(n-(n+m)) t+\frac{\frac{1}{r}}{t^{\frac{n-(n+m)}{n+m}}}(n+m)\right] e^{i a} \\
& =r\left[n-(n-m) \tau^{\frac{n+m}{n-(n+m)}}+\frac{(n+m) \frac{1}{r}}{\tau}\right] e^{i a} \\
& =r \operatorname{rymap}\left[\tau, n, m+n, \frac{1}{r}, a\right]
\end{aligned}
$$

(ii) Let hymap $[t, n, m, r, a]=\left[(n-m) t^{\frac{m}{n-m}}+\frac{r m}{t}\right] e^{i a}$ and $\tau=t^{\frac{m}{n-m}}$. Then $t=\tau^{\frac{n-m}{m}}$. We get

$$
\begin{aligned}
\text { hymap }[t, n, m, r, a] & =\left[(n-m) t^{\frac{m}{n-m}}+\frac{r m}{t}\right] e^{i a} \\
& =r\left[\frac{(n-m)}{r} t^{\frac{m}{n-m}}+\frac{m}{t}\right] e^{i a} \\
& =r\left[\frac{n+(m-n)}{t}-\frac{(m-n)}{r} t^{\frac{m}{n-m}}\right] e^{i a} \\
& =r\left[\frac{n+(m-n)}{t}-(m-n) \frac{1}{r} t^{\frac{n+(m-n)}{n-m}}\right] e^{i a} \\
& =r\left[n+(n-m) \tau^{\frac{m-n}{m}}-(m-n) \frac{1}{r} \tau\right] e^{i a} \\
& =r \operatorname{epmap}[\tau, n, m-n, 1 / r, a]
\end{aligned}
$$

The B-E-G theorem comes into play in the design of the rotary engine, where the bore is in the shape of an epitrochoid, and the rotor is determined using the equivalent hypotrochoid. The center of the rotor traces out a circle, which corresponds to the driveshaft. A nice Mathematica animation can be made of a working rotary engine, complete with rotating driveshaft. 
To illustrate the B-E-G Theorem, we shall plot the graphs of hymap $[t, 7,3,1 / 2,0]$ and $1 / 2$ epmap $[\tau, 7,-4,2,0]$, where we use part (ii) with $n=7, m=3, r=1 / 2$, and $a=0$. The plots will be identical.

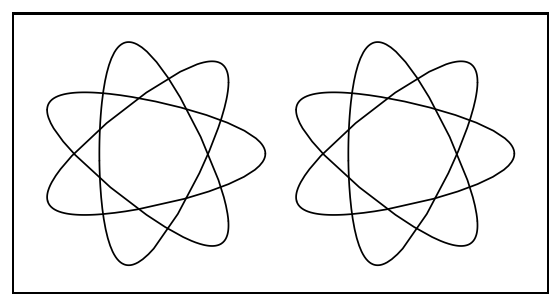

Fig. 3: 2 hymap $[t, 7,3,1 / 2,0]=\operatorname{epmap}[\tau, 7,-4,2,0]$.

Theorem 3. (The Complement Theorem) If $(n, m)=1$, then

$$
\text { hymap }[t, n, m, r, a]=\operatorname{rhymap}\left[\tau, n, n-m, \frac{1}{r}, a\right]
$$

where $\tau=t^{\frac{m}{m-n}}$

Proof. Using $\tau=t^{\frac{m}{m-n}}$, we get $t=\tau^{\frac{m-n}{m}}$. Then

$$
\begin{aligned}
\text { hymap }[t, n, m, r, a] & =\left[(n-m) t^{\frac{m}{n-m}}+\frac{r m}{t}\right] e^{i a} \\
& =r\left[\frac{(n-m)}{r} t^{\frac{m}{n-m}}+\frac{m}{t}\right] e^{i a} \\
& =r\left[\frac{n-(n-m)}{t}+\frac{(n-m) \frac{1}{r}}{t^{\frac{m}{m-n}}}\right] e^{i a} \\
& =r\left[(n-(n-m)) \tau^{\frac{n-m}{n-(n-m)}}-\frac{(n-m) \frac{1}{r}}{\tau}\right] e^{i a} \\
& =r \text { hymap }\left[\tau, n, n-m, \frac{1}{r}, a\right]
\end{aligned}
$$

For example, hymap $[t, 7,1,1,0]$ and hymap $[\tau, 7,6,1,0]$ both produce the following graph, the curve being traced out counterclockwise when $m=1$ and clockwise when $m=6$. Such congruences follow from the Complement theorem because the radius of the two rolling circles sum to $n$. Another connection between epitrochoids and hypotrochoids, which can be proved either by appealing to the definitions or by applying the Complement and B-E-G theorems, is that epmap $[t, n, m, r, a]=$ hymap $[\tau, n,-m, r, a]$. Using $t=\tau^{-1}$

$$
\begin{aligned}
\operatorname{epmap}[t, n, m, r, a] & =\left[(n+m) t^{\frac{m}{n+m}}-r m t\right] e^{i a} \\
& =\left[(n-(-m)) t^{\frac{n-(n-m)}{n-(-m)}}+r(-m) t\right] e^{i a} \\
& =\left[(n-(-m)) \tau^{\frac{-m}{n+m}}+\frac{r(-m)}{\tau}\right] e^{i a} \\
& =\operatorname{hymap}[\tau, n,-m, r, a] .
\end{aligned}
$$

We now look at the idea of multiple points of trochoids. A $n$-fold point of a trochoid is a point that is traced over exactly $n$ times during one fundamental period. A rose with $n$ petals has an $n$-fold point at $(0,0)$. The next theorem gives a necessary and sufficient condition for the hypotrochoid with second argument $n$ to have an $n$-fold point at $(0,0)$. We shall call this kind of hypotrochoid, as well as any other curve with $n$ "petals" and an $n$-fold point at $(0,0)$ a rosette. First, however, we need a theorem. 


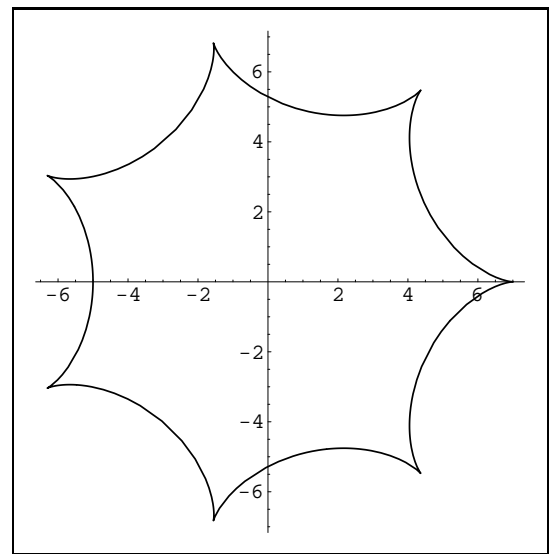

Fig. 4: hymap[t,7,1,1,0] and hymap $[\tau, 7,6,1,0]$.

Theorem 4. The graphs of hymap $[t, n, m,-r, a+m \pi / n]$ and hymap $[t, n, m, r, a]$ are congruent.

Proof. A proof based on the definitions is possible here, but geometric reasoning is more intuitive. It is easier to picture what is going on if $m<n$, but the lemma remains true if $m \geq n$. Geometrically, $t$ is the counterclockwise angle through which the center of the rolling circle has moved, measured from the starting, angle $a$, and $\mathrm{m} \pi / \mathrm{n}$ is the $t$-increment corresponding to half of one revolution of the rolling circle. Thus, when $t=m \pi / n$, the position of the rolling circle in hymap $[t, n, m, r, a]$ is: center at angle $(a+m \pi / n)$ and tracing point diametrically opposite its starting point with respect to the rolling circle. But this is the same as the starting position for hymap $[t, n, m,-r, a+m \pi / n]$.

Theorem 5. (The Rosette Theorem I) If $(n, m)=1$, hymap $[t, n, m, r, a]$ is a rosette with $n$-fold point at $(0,0)$ if and only if $r=\left(\frac{n-m}{m}\right)$.

Proof. ( $\Longrightarrow$ ) Suppose hymap[t,m,n,r,a] is a rosette with n-fold point at $(0,0)$. Then

$$
\text { hymap }[t, n, m, r, a]=\left[(n-m) t^{\frac{m}{n-m}}+\frac{r m}{t}\right] e^{i a}
$$

and there are exactly $n$ turns $t$ such that

$$
0=\left((n-m) t^{\frac{m}{n-m}}+\frac{m r}{t} e^{i a}\right.
$$

which is equivalent to

$$
t^{\frac{n}{n-m}}=\frac{m r}{m-n} .
$$

Because $t$ is a turn,

$$
\begin{aligned}
\left|t^{n}\right| & =\left|t^{\frac{n}{n-m}}\right|=1, \\
1 & =\left|\frac{m r}{m-n}\right|, \\
r & = \pm \frac{n-m}{m} .
\end{aligned}
$$

$(\Longleftarrow)$ Suppose $r= \pm \frac{n-m}{m}$. Then $r m= \pm(n-m)$.

Case 1. Suppose $r m=n-m$. 


$$
\begin{aligned}
\operatorname{hymap}[t, n, m, r, a] & =\operatorname{hymap}\left[t, n, m, \frac{n-m}{m}, a\right] \\
& =\left[(n-m) t^{\frac{m}{n-m}}+\frac{(n-m)}{t}\right] e^{i a} \\
& =\left[(n-m)\left[t^{\frac{m}{n-m}}+\frac{1}{t}\right]\right] e^{i a} \\
& =\left[(n-m)\left[\frac{t^{\frac{n}{n-m}}+1}{t}\right]\right] e^{i a} .
\end{aligned}
$$

The curve passes through $(0,0)$ when $t=\left[(-1)^{n-m}\right]^{\frac{1}{n}}$ and because these are $n^{\text {th }}$ root of either 1 or -1 , there are exactly $\mathrm{n}$ of them in one circuit of the unit circle.

Case 2. Suppose $r m=-(n-m)$. In the same way,

$$
\operatorname{hymap}[t, n, m, r, a]=\left[(n-m)\left[\frac{t^{\frac{n}{n-m}}-1}{t}\right]\right] e^{i a}
$$

The curve passes through $(0,0)$ when $t=\left[1^{n-m}\right]^{\frac{1}{n}}$, an $n^{t h}$ root of unity, of which there are exactly $\mathrm{n}$ in one circuit of the unit circle.

To illustrate the Theorem, here is the graph of $h y[t, 12,1,11,0]$ (Figure 5). The theorem conditions for a 12-fold point at $(0,0)$ are satisfied.

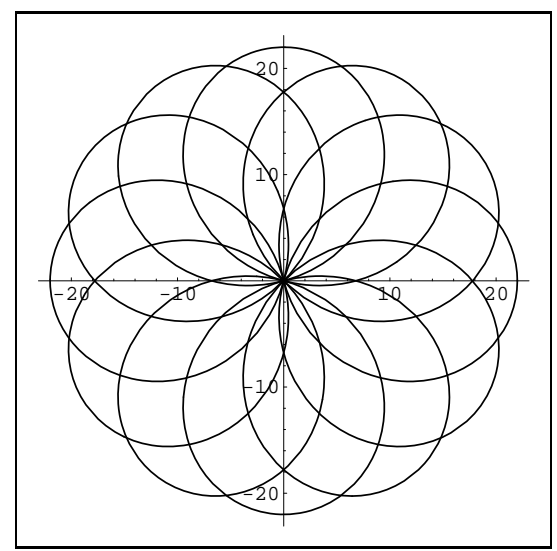

Fig. 5: hymap[t,12,1,11,0].

Definition 8. For a rosette with map equation hymap $[t, n, m, r, a],($ or epmap $[t, n, m, r, a])$, if hymap $\left[\tau_{1}, n, m, r, a\right]=$ hymap $\left[\tau_{2}, n, m, r, a\right]=0$ and there are no other zeros between $\tau_{1}$ and $\tau_{2}$, then that part of the rosette corresponding values of t between $\tau_{1}$ and $\tau_{2}$ is a petal. A petal angle is the angle between the tangents to a petal at the origin.

In other words, a petal is one loop of the rosette. By the symmetry, if $a=0$, every kind of petal intersection will occur on either the $\mathrm{x}$-axis or the line with polar angle $\frac{\pi}{n}$. If $a \neq 0$, the intersections are rotated by the angle $a$.

Theorem 6. (Petal Angle Theorem) The petal angle of a rosette is

$$
\alpha=\left|\operatorname{Re}\left\{1 \log \left(-(-1)^{\frac{-2 m}{n}}\right)\right\}\right| .
$$


Proof. From Rosette Theorem I, $r=\frac{n-m}{m}$ or $r m=n-m$. Thus, in the hypocycloid map equation, we get

$$
\text { hymap }[t, n, m, r, a]=\left((n-m) t^{\frac{m}{n-m}}+\frac{n-m}{t}\right) e^{i a}=\left[(n-m)\left[t^{\frac{m}{n-m}}+\frac{1}{t}\right] e^{i a}\right.
$$

Let $t=\tau^{n-m}$. Then $t^{\frac{m}{n-m}}=\tau^{m}$. Using this assumption above, we get

$$
\text { hymap }[\tau, n, m, r, a]=(n-m)\left[\tau^{m}+\frac{1}{\tau^{n-m}}\right] e^{i a}=(n-m) \tau^{m-n}\left(1+\tau^{n}\right) e^{i a} .
$$

We want to find the petal angle of the hypocycloid. Using the definition of petal angles, we need consecutive turns where hymap $[t, n, m, r, a]=0$. That means $\tau^{n}+1=0$, and $\tau=(-1)^{\frac{1}{n}}$. Differentiating, we get

$$
D_{\tau} \text { hymap }=(n-m)\left[m \tau^{m-1}+(m-n) \tau^{m-(n+1)}\right] e^{i a},
$$

and

$$
\overline{D_{\tau} \text { hymap }}=(n-m)\left[m \tau^{1-m}+(m-n) \tau^{n+1-m}\right] e^{i a} .
$$

Hence, we can obtain the clinant of the tangent from the map equation,

$$
c=\frac{\overline{D_{\tau} \text { hymap }}}{\tau^{2} D_{\tau} \text { hymap }}=\frac{(n-m)\left[m \tau^{1-m}+(m-n) \tau^{n+1-m}\right] e^{i a}}{\tau^{2}(n-m)\left[m \tau^{m-1}+(m-n) \tau^{m-(n+1)}\right] e^{i a}}=\frac{\tau^{1-m}\left[(m-n) \tau^{n}+m\right]}{\tau^{2}\left(\tau^{m-1}\left[(m-n) \tau^{-n}+m\right]\right)} .
$$

Since this is to be evaluated where $\tau^{n}=-1$ and $\tau^{-n}=-1$,

$$
c=\frac{1}{\tau^{2 m}} .
$$

Because $\tau=(-1)^{\frac{1}{n}}$,

$$
c=(-1)^{\frac{-2 m}{n}} .
$$

The inclination angle $\phi=\frac{i}{2} \log \left(-(-1)^{\frac{-2 m}{n}}\right)$. Therefore

$$
\alpha=2|\phi|=2\left|\frac{i}{2} \log \left(-(-1)^{\frac{-2 m}{n}}\right)\right|=\left|i \log \left(-(-1)^{\frac{-2 m}{n}}\right)\right|=\left|\operatorname{Re}\left\{i \log \left(-(-1)^{\frac{-2 m}{n}}\right)\right\}\right|
$$

Theorem 7. (Petal Angle Sum Theorem) The sum of all the petal angles of the trochoid hymap $[t, n, m, r, a]$ is

$$
n \alpha=|n-2 m| \pi
$$

Proof.Case 1. If $n=2 k$, then

$$
\begin{aligned}
n \alpha & =2 k\left|\operatorname{Re}\left\{i \log \left(-(-1)^{\frac{-2 m}{2 k}}\right)\right\}\right| \\
& =2 k\left|\operatorname{Re}\left\{i \log \left(e^{i \pi}\left(e^{i \pi}\right)^{\frac{-m}{k}}\right)\right\}\right| \\
& =2 k \mid \operatorname{Re}\left\{-\pi\left(1-\frac{m}{k}\right\} \mid\right. \\
& =2 k \pi\left|1-\frac{m}{k}\right| \\
& =\pi|2 k-2 m| \\
& =\pi|n-2 m|
\end{aligned}
$$

Cese 2. If $n=2 k+1$, then 


$$
\begin{aligned}
n \alpha & =(2 k+1)\left|\operatorname{Re}\left\{i \log \left(-(-1)^{\frac{-2 m}{2 k+1}}\right)\right\}\right| \\
& =(2 k+1) \mid \operatorname{Re}\left\{i \log \left(e^{i \pi}\left(e^{i \pi}\right)^{\frac{-2 m}{2 k+1}}\right\} \mid\right. \\
& =(2 k+1) \mid \operatorname{Re}\left\{-\pi\left(1-\frac{2 m}{2 k+1}\right\} \mid\right. \\
& =\left|(2 k+1)-\pi\left(1-\frac{2 m}{2 k+1}\right)\right| \\
& =\pi\left|(2 k+1)-(2 k+1) \frac{2 m}{2 k+1}\right| \\
& =\pi|n-2 m|
\end{aligned}
$$

Theorem 8. (The Rose Theorem) If $(n, m)=1$, the hypotrochoid hymap $[t, n, m, r, a]$ is a rose if and only if

$$
r= \pm \frac{(n-m)}{m} \text { and either } m=\left[\frac{n}{2}\right]+1 \text { or } m=n-\left[\frac{n}{2}\right]-1
$$

where $\left[\frac{n}{2}\right]$ denotes the greatest integer in $\frac{n}{2}$.

Proof. ( $\Longrightarrow$ Suppose hymap $[t, n, m, r, a]$ is a rose. Then hymap $[t, n, m, r, a]$ is a rosette, and $r= \pm \frac{m-n}{n}$. The sum of the petal angles of a rose is $\pi$ or $2 \pi$. That means $n-2 m$ is \pm 1 or \pm 2 .

Case 1. Suppose $n$ is odd. Then $\left[\frac{n}{2}\right]=\frac{n-1}{2}$. Since $n$ is odd, $n-2 m$ must be equal to \pm 1 . Suppose $n-2 m=1$. Then

$$
m=\frac{n-1}{2}=n-\frac{n-1}{2}-1=n-\left[\frac{n}{2}\right]-1 .
$$

Suppose $n-2 m=-1$. Then

$$
m=\frac{n+1}{2}=\frac{n-1}{2}+1=\left[\frac{n}{2}\right]+1
$$

Case 2. Suppose $n$ is even. Then $\left[\frac{n}{2}\right]=\frac{n}{2}$. Since $\mathrm{n}$ is even, $n-2 m$ must be equal to \pm 2 .

Suppose $n-2 m=2$. Then

$$
m=\frac{n-2}{2}=n-\frac{n}{2}-1=n-\left[\frac{n}{2}\right]-1 .
$$

Suppose $n-2 m=-2$. Then

$$
m=\frac{n+2}{2}=\frac{n}{2}+1=\left[\frac{n}{2}\right]+1
$$

( $\Longleftarrow$ Suppose $r= \pm \frac{(n-m)}{m}$. Then the curve is a rosette.

Case 1. Suppose $n$ is odd and $m=\left[\frac{n}{2}\right]+1$. Then $\left[\frac{n}{2}\right]=\frac{n-1}{2}$ and $m=\frac{n+1}{2}$. The sum of the petal angles of the rosette is

$$
\begin{aligned}
|n-2 m| \pi & =|n-(n+1)| \pi \\
& =\pi .
\end{aligned}
$$

Case 2. Suppose $n$ is odd and $m=n-\left[\frac{n}{2}\right]-1$. Then $\left[\frac{n}{2}\right]=\frac{n-1}{2}$ and $m=\frac{n-1}{2}$. The sum of the petal angles of rosette is

$$
|n-2 m| \pi=|n-(n-1)| \pi=\pi
$$

The sum of the petal angles is $\pi$ in case(i) and case (ii). Therefore, the curve has no double point and hymap $[t, n, m, r, 0]$ is a rose.

Case 3. Suppose $n$ is even and $m=\left[\frac{n}{2}\right]+1$. Then $\left[\frac{n}{2}\right]=\frac{n}{2}$ and $m=\frac{n+2}{2}$. The sum of the petal angles of the rosette is 


$$
|n-2 m| \pi=|n-(n+2)| \pi=2 \pi .
$$

Case 4. Suppose $n$ is even and $m=n-\left[\frac{n}{2}\right]-1$. Then $\left[\frac{n}{2}\right]=\frac{n}{2}$ and $m=\frac{n-2}{2}$. The sum of the petal angles of the rosette is

$$
|n-2 m| \pi=|n-(n-2)| \pi=2 \pi .
$$

The sum of the petal angles is $2 \pi$ in case(iii) and case (iv). Therefore, the curve has no double point and hymap $[t, n, m, r, 0]$ is a rose.

Theorem 9. (The Rosette Theorem II) If $(n, m)=1$, the curve defined by

$$
d m a p\left[t, n, m, r_{1}, r_{2}, a\right]=\operatorname{epmap}\left[t, n, m, r_{1}, a\right]-\operatorname{hymap}\left[t, n, m, r_{2}, a\right]
$$

is a rosette of $n$ petals if and only if $\left|r_{1}+r_{2}\right|=2$.

Proof. First, we will combine the map equations.

$$
\begin{aligned}
\operatorname{dmap}\left[t, n, m, r_{1}, r_{2}, a\right] & =\left((n+m) t-r_{1} m t^{\frac{n+m}{m}}\right)-\left((n-m) t+\frac{m r_{2}}{t^{\frac{n-m}{m}}}\right) e^{i a} \\
& =\left(2 m t-r_{1} m t^{\frac{n+m}{m}}-\frac{m r_{2}}{t^{\frac{n-m}{m}}}\right) e^{i a} \\
& =\left[\frac{-m}{t^{\frac{n-m}{m}}}\left(-2 t^{\frac{n}{m}}+r_{1} t^{\frac{2 n}{m}}+r_{2}\right)\right] e^{i a} .
\end{aligned}
$$

Let $t^{1 / m}=\tau$. Then we get the complete equation:

$$
\operatorname{dmap}\left[\tau, n, m, r_{1}, r_{2}, a\right]=\left[\frac{-m}{\tau^{n-m}}\left(r_{1} \tau^{2 n}-2 \tau^{n}+r_{2}\right)\right] e^{i a} .
$$

For convenience, we will write $\operatorname{dmap}(\tau)$ instead of $\operatorname{dmap}\left[\tau, n, m, r_{1}, r_{2}, a\right]$ in the rest of the proof.

$\Leftrightarrow$ If $\operatorname{dmap}(\tau)$ is a rosette of $n$ petals, then there are exactly $n$ turns $\tau$ for which $\operatorname{dmap}(\tau)=0$. If $\operatorname{dmap}(\tau)=0$, then

$$
\tau^{n}=\frac{1 \pm \sqrt{1-r_{1} r_{2}}}{r_{1}}
$$

So for one sign choice, but not both, $\left|\tau^{n}\right|=1$. There are three cases:

(i) $r_{1} r_{2}<1: \tau^{n}=\frac{1}{r_{1}}\left(1 \pm \sqrt{1-r_{1} r_{2}}\right)>0$ and $\tau^{n}$ is real.

Let $\tau^{n}=1$. Then

$$
\begin{aligned}
\frac{1}{r_{1}}\left(1 \pm \sqrt{1-r_{1} r_{2}}\right) & =1 \\
1 \pm \sqrt{1-r_{1} r_{2}} & =r_{1} \\
1-r_{1} r_{2} & =\left(r_{1}-1\right)^{2} \\
r_{1}^{2}-r_{1}\left(2-r_{2}\right) & =0 .
\end{aligned}
$$

Therefore $r_{1}=0$ or $r_{1}=2-r_{2}$. If $r_{1}=0$, then $\tau^{n}=\frac{r_{2}}{2}$ so $r_{2}=2$, and if $r_{1}=2-r_{2}$, then $r_{1}+r_{2}=2$.

If $\tau^{n}=-1$ similar work gives $r_{1}+r_{2}=-2$.

(ii) If $r_{1} r_{2}=1$, then $\tau^{n}=\frac{1}{r_{1}}$ and $\left|\tau^{n}\right|=1$. If $r_{1}= \pm 1$, then $r_{2}= \pm 1$ and $r_{1}+r_{2}= \pm 2$.

(iii) Suppose $r_{1} r_{2}>1$. Then $\tau^{n}=\frac{1}{r_{1}} \pm i \frac{\sqrt{r_{1} r_{2}-1}}{r_{1}}$ and $\left|\tau^{n}\right|^{2}=\frac{r_{2}}{r_{1}}$. But, to be a turn, we need $\left|\frac{r_{2}}{r_{1}}\right|=1$. Then $r_{1}=r_{2}$ or $r_{1}=-r_{2}$. The case $r_{1}=-r_{2}$ is impossible because $r_{1} r_{2}>1$ fails. So $\operatorname{dmap}(\tau)=0$ in this case only if $r_{1}=r_{2}= \pm 1$. 


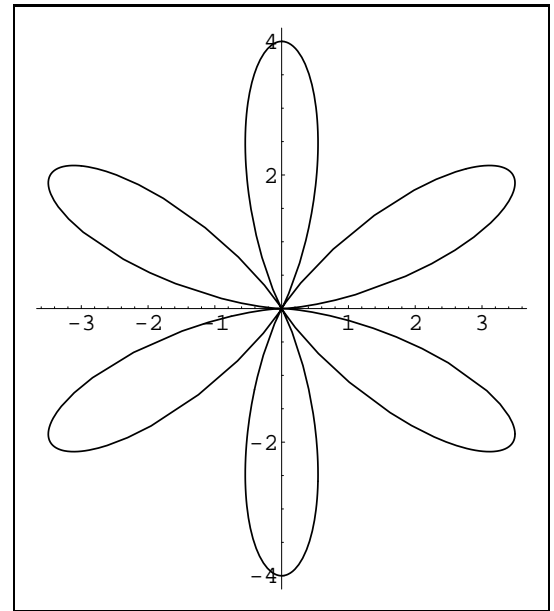

Fig. 6: dmap $[\mathrm{t}, 6,1,1,1,0]=e p m a p[t, 6,1,1,0]$-hymap $[\mathrm{t}, 6,1,1,0]$.

$(\Leftarrow)$ If $r_{1}+r_{2}=2$, then $\operatorname{dmap}(\tau)=\frac{-m}{\tau^{n-m}}\left(\tau^{n}-1\right)\left(r_{1} \tau^{n}-r_{2}\right)$, and if $r_{1}+r_{2}=-2$, then $d m a p(\tau)=\frac{-m}{\tau^{n-m}}\left(\tau^{n}+1\right)\left(r_{1} \tau^{n}+r_{2}\right)$. In either case, the only solution of $\operatorname{dmap}(\tau)$ which is a turn is $\tau^{n}= \pm 1$, and the origin has an n-fold point.

Let's consider dmap $[t, 6,1,1,1,0]=\operatorname{epmap}[t, 6,1,1,0]-$ hymap $[t, 1,1,1,0]$. After we combine the map equations, we will get

$$
\operatorname{dmap}[t, 6,1,1,1,0]=\frac{-1}{t^{5}}\left(-2 t^{6}+t^{32}+1\right) .
$$

Because $m=1$, this is already in the conjugate form. When we look at the graph of the dmap the roots are double roots and the curve to "bounce back" at $(0,0)$ instead of "passing through".

Corollary 1. If $(n, m)=1$, the curve defined by

$$
\operatorname{dmap}[t, n, m, r, a]=\operatorname{epmap}[t, n, m, r, a]-\operatorname{hymap}[t, n, m, r, a]
$$

is a rosette with a $2 n-$ fold point at the origin if and only if $|r|>1$.

Actually, the rosettes in Rosette Theorem II include those in Rosette Theorem I, and thus also include roses. The relationship is given in the following corollary. A similar result holds with -2 in place of 2 .

\section{Corollary 2.}

$$
\operatorname{dmap}[\tau, n, m, 0,2, a]=\frac{2 m}{(n-m)} \operatorname{hymap}\left[\tau, n, m,-\frac{n}{m}+1, a\right]
$$

\section{Conclusion}

Mathematical modelling regarding to algebraic curves is a long studied subject. In this study, trochoids and related curves using the representation of these curves as mapping of the unit circle in the complex plane is given. First we define the map equation of these curves with the self-conjugate relations. Then, we give the conditions for trochoids to be complete. Since map equations are simpler than parametric equations, we introduce the basic results on trochoids and epitrochoids by using their map equations. A classical result regarding trochoids is that any hypotrochoid can be expressed in terms of an epitrochoid and viceversa. To obtain this result, we prove the Bernoulli - Euler- Goldbach double generation theorem on map equations. We also present a connection between epitrochoids and hypotrochoids by applying the Complement and B-E-G theorems, is that epmap $[t, n, m, r, a]=h y m a p[t, n,-m, r, a]$. We also present the relationships between the hypotrochoids and rosettas by using map equations. 


\section{Competing interests}

The authors declare that they have no competing interests.

\section{Authors' contributions}

All authors have contributed to all parts of the article. All authors read and approved the final manuscript.

\section{References}

[1] Abel, U., Beukemann, L., \& Kushnirevych, V. (2017). Rolling Curves: An Old Proof of the Roulette Lemma. The American Mathematical Monthly, 124(8), 723-736.

[2] Berendonk, S. (2017). Double generating spirographic curves. The Mathematical Gazette, 101(550), 27-37.

[3] Farrington, E. (2015). Parametric Equations at the Circus: Trochoids and Poi Flowers. The College Mathematics Journal, 46(3), 173-177.

[4] Hall, L. (1992). Trochoids, roses, and thorns - beyond Spinographs. College Mathematics Journal, 23(1), 20-35.

[5] Moritz, R. E. (1917). On the Construction of Certain Curves Given in Polar Coordinates. The American Mathematical Monthly, 24(5), 213-220.

[6] Rigge, W. F. (1918). A compound harmonic motion machine I, II. Scientific American Supplement, 2197(2198), 88-91.

[7] Rigge, W. F. (1919). Discussions: Concerning a New Method of Tracing Cardioids. The American Mathematical Monthly, 26(1), 21-32.

[8] Rigge, W. F. (1919). Cuspidal rosettes. The American Mathematical Monthly, 26(8), 332-340.

[9] Rigge, W. F. (1920). Envelope rosettes. The American Mathematical Monthly, 27(4), 151-157.

[10] Rigge, W. F. (1922). Cuspidal envelope rosettes. The American Mathematical Monthly, 29(1), 6-8.

[11] Tsiotras, P., \& Reyes Castro, L. I. (2010). A note on the consensus protocol with some applications to agent orbit pattern generation. Georgia Institute of Technology. 